\title{
The use of metformin to reduce metabolic complications and inflammation in patients on systemic glucocorticoid therapy: a randomised, double-blind, placebo-controlled, phase 2 clinical trial
}

\author{
Short title: Metformin to reduce metabolic complications of systemic glucocorticoids: RCT \\ Ida Pernicova ${ }^{1,2} \mathrm{MD} / \mathrm{PhD}$, Stephen Kelly ${ }^{3} \mathrm{MD} / \mathrm{PhD}$, Sharon Ajodha ${ }^{1}$, Anju Sahdev ${ }^{4} \mathrm{MD}$, Jonathan P Bestwick ${ }^{5}$ \\ MSc, Plamena Gabrovska ${ }^{1} \mathrm{PhD}$, Akanle Olufunso ${ }^{4} \mathrm{PhD}$, Ramzi Ajjan ${ }^{6} \mathrm{MD} / \mathrm{PhD}$, Blerina Kola $\mathrm{MD} / \mathrm{PhD}$, \\ Marietta Stadler ${ }^{1,7} \mathrm{MD}$, William Fraser ${ }^{8} \mathrm{MD} / \mathrm{PhD}$, Mirjam Christ-Crain ${ }^{1,9} \mathrm{MD} / \mathrm{PhD}$, Ashley B Grossman $\mathrm{MD}^{1}$, \\ Costantino Pitzalis ${ }^{3} \mathrm{MD} / \mathrm{PhD}$, Márta Korbonits ${ }^{1} \mathrm{MD} / \mathrm{PhD}$
}

${ }^{1}$ Centre for Endocrinology, William Harvey Research Institute, Barts and the London School of Medicine and Dentistry, Queen Mary University of London, London EC1M 6BQ, UK

${ }^{2}$ Endocrinology and Metabolic Medicine, Sheffield Teaching Hospitals NHS Foundation Trust, Sheffield S10 2JF, UK

${ }^{3}$ Centre for Experimental Medicine \& Rheumatology, William Harvey Research Institute, Barts and the London School of Medicine and Dentistry, Queen Mary University of London, London EC1M 6BQ, UK

${ }^{4}$ Department of Radiology, Barts Health NHS Trust, London EC1A 7BE, UK

${ }^{5}$ Wolfson Institute of Preventive Medicine, Barts and the London School of Medicine and Dentistry, Queen Mary University of London, London EC1M 6BQ, UK

${ }^{6}$ Leeds Institute of Cardiovascular and Metabolic Medicine, University of Leeds, Leeds LS2 9JT, UK

${ }^{7}$ King's College London, Faculty of Life Sciences and Medicine, Department of Diabetes, Denmark Hill Campus, London SE5 9RJ, UK

${ }^{8}$ University of East Anglia, Norwich NR4 7TJ, UK

${ }^{9}$ Division of Endocrinology, Diabetes \& Metabolism, Department of Internal Medicine and Department of

Clinical Research, University Hospital, University of Basel, Basel CH-4031, Switzerland

Word Number: 4120 (main text)

Number of Figures 3; Number of Tables 3, BOX - Research in context

Supplementary material: 2 figures and 12 tables

Corresponding author:

Professor Márta Korbonits

Centre for Endocrinology, William Harvey Research Institute Queen Mary University of London, Charterhouse Square, London EC1M 6BQ, UK

Phone: +44 2078826238

Fax: +44 2078826197

Email: m.korbonits@qmul.ac.uk 


\begin{abstract}
Background: There is an urgent need to limit the metabolic side-effects of glucocorticoid overexposure as these can lead to Cushing's syndrome, associated with high morbidity. We have explored the potential for metformin to ameliorate such effects whilst sparing the anti-inflammatory benefits of glucocorticoids.
\end{abstract}

Methods: In this double-blind, phase 2 proof-of-concept trial, 53 patients without known diabetes established on mid-to-high doses of glucocorticoids, administered as treatment for a chronic inflammatory disease, were randomised to receive $2550 \mathrm{mg} /$ day metformin $(n=26)$ or an identical placebo $(n=27)$ for 12 weeks. The primary endpoint was the change in visceral to truncal subcutaneous fat ratio assessed by computed tomography; secondary endpoints involved metabolic, bone, cardiovascular and inflammatory parameters.

Findings: Nineteen patients on metformin and 21 on placebo completed the study. The groups received equivalent cumulative dose of glucocorticoids (1860mg (IQR 1060 to 2810) vs. 1770mg (IQR 1020-2356)

prednisolone equivalent; $\mathrm{p}=0.76)$. There was no change in the visceral-to-subcutaneous fat ratio $(0.11(95 \% \mathrm{CI}$ 0.02 to 0.24$) ; \mathrm{p}=0.09$ ) between the treatment groups but metformin-treated patients lost truncal subcutaneous fat $\left(-3835 \mathrm{~mm}^{2}(95 \% \mathrm{CI}-6781\right.$ to -888$\left.) ; \mathrm{p}=0.01\right)$ compared to placebo. Improvements in markers of carbohydrate, lipid, liver and bone metabolism were observed on metformin. Additionally, metformin-treated patients had improved fibrinolysis, carotid intima-media thickness, inflammatory parameters and clinical markers of disease activity. The frequency of pneumonia ( 1 vs. 7 events; $p=0.01)$, overall rate of moderate-to-severe infections $(2$ vs. $11 ; \mathrm{p}=0.001)$, and all-cause hospital admissions due to adverse events ( 1 vs. $9 ; \mathrm{p}=0.001)$ were lower in the metformin group compared with placebo. Metformin-treated patients experienced more diarrhoea initially.

Interpretation: Metformin administration improved the metabolic profile of glucocorticoid-treated patients with inflammatory disease, favourably modifying cardiovascular risk surrogates, reducing inflammation and hospitalisation.

Funding: Barts Charity, Merck Serono. ClinicalTrials.gov NCT01319994. 


\section{Research in context}

\section{Evidence before this study}

Glucocorticoid excess, due to endogenous causes or to glucocorticoid treatment, can lead to Cushing's syndrome, a phenotype associated with an increased morbidity and mortality. The higher morbidity risk lasts even after removal of glucocorticoid excess.

Our team have previously shown that several metabolic changes associated with glucocorticoid overexposure correspond to metabolic steps regulated by 5'AMP-activated-protein-kinase (AMPK). AMPK is one of the mediators of metformin's action: metformin was able to reverse the glucocorticoid effect on AMPK in vitro and to prevent glycaemic deterioration in non-diabetic patients when initiated simultaneously with glucocorticoid treatment.

Metformin treatment was shown to be associated with metabolic benefits on weight, blood pressure, lipids, coagulation, inflammation, and endothelium in some, but not all, human studies. We wondered whether metformin administration for patients with glucocorticoid treatment might result in beneficial metabolic effects without adversely affecting their anti-inflammatory actions.

\section{Added value of this study}

This randomised, double-blind, placebo-controlled, phase 2 clinical trial evaluated patients established on glucocorticoid treatment, without diabetes, for the potential beneficial effects of metformin to diminish multisystem adverse effects of glucocorticoid treatment. Our data do not just show improvement in several surrogates of metabolic and cardiovascular risks for patients receiving metformin, but the intervention also appeared to reduce inflammation and number of infections and hospitalisation of this vulnerable patient cohort. The results are clinically relevant, and could be applied efficiently and economically to a large number of patients worldwide.

\section{Implications of all the available evidence}

Although glucocorticoids are the most common cause of medication-induced hyperglycaemia, and rising glycaemia has been reported as the number one concern when prescribing glucocorticoid treatment, there is no international consensus on the screening and management of glucocorticoid-induced hyperglycaemia. Based on experimental data, metformin's mode of action may interact with the glucocorticoid pathway. We propose that metformin, irrespective of the diabetic status, could be a good agent to attenuate adverse effects of glucocorticoid treatment, reducing inflammation and cost, and improving patient outcomes. In view of limitations of lifestyle intervention for patients in need of glucocorticoid therapy, based on this study we recommend patients be considered for concomitant metformin treatment early and that larger studies are initiated to evaluate hard cardiovascular endpoints. 


\section{INTRODUCTION}

Glucocorticoid treatment is prescribed long-term in up to $3 \%$ of the adult population ${ }^{1,2}$. Chronic exposure to glucocorticoid excess can lead to distinctive truncal obesity, hypertension, hyperglycaemia, dyslipidaemia, hypercoagulability, fatty liver, osteoporosis, increased infections and other complications ${ }^{3}$. The developing phenotype, known as iatrogenic Cushing's syndrome, is associated with increased morbidity and mortality, especially from cardiovascular causes and infections ${ }^{3,4}$ and the raised morbidity persists even after abrogation of glucocorticoid excess ${ }^{5,6}$. Separating the desired anti-inflammatory effects of glucocorticoids from their unwanted metabolic action has so far proved difficult.

In patients with Cushing's syndrome, the activity of one of the enzymatic mediators of metformin's action, 5'AMP-activated-protein-kinase (AMPK) ${ }^{7}$, was decreased in visceral adipose tissue ${ }^{8}$ while metformin reversed the glucocorticoid effect on AMPK in adipose and hypothalamic tissues in vitro ${ }^{9}$, and improved glucose levels in patients who were starting glucocorticoid treatment ${ }^{10}$. Metformin was also associated with a favourable immune response in several animal models of autoimmune diseases ${ }^{7}$. We hypothesised that metformin might alleviate a plethora of metabolic features of glucocorticoid overexposure without adversely affecting their antiinflammatory benefits. Therefore, we designed a randomised, double-blind, placebo-controlled, phase 2 proofof-concept trial of the addition of metformin therapy in patients with chronic inflammatory diseases already established on long-term glucocorticoid treatment. We wished to evaluate the potential of metformin to reverse multisystem glucocorticoid metabolic side-effects. There is clearly an urgent need for such a resolution but no current solution. We aimed to improve the metabolic profile associated with glucocorticoid treatment and demonstrate that metabolically vulnerable patients with substantial cumulative glucocorticoid exposure significantly benefited from the administration of metformin.

\section{METHODS}

\section{Participants}


Eligible patients in this randomised, double-blind, placebo-controlled, phase 2 proof-of-concept trial were adults ( $\geq 18$ and $\leq 75$ years) with an inflammatory disease with ongoing $\geq 20 \mathrm{mg} /$ day prednisolone treatment for $\geq 4$ weeks (or its cumulative dose-equivalent), remaining on $\geq 10 \mathrm{mg} /$ day prednisolone-equivalent for $\geq 12$ subsequent weeks. Cumulative prednisolone equivalent was calculated using recognized conversion tables and both oral and parenteral glucocorticoids were taken into account. Minimum duration of continuous glucocorticoid exposure prior enrolment was 4 weeks, minimum cumulative dose: 560mg prednisolone equivalent. Key exclusion criteria were known pre-existing diabetes mellitus, prior therapy with metformin over the previous 6 months, ALT and/or AST $\geq 2.5 \times$ upper limit of normal, or serum creatinine $\geq 135 \mu \mathrm{mol} / \mathrm{L}$ (males) and $\geq 110 \mu \mathrm{mol} / \mathrm{L}$ (females) (further details: Supplementary Methods).

\section{Study design, randomisation and masking}

After assessing 849 patients attending respiratory and rheumatology outpatient clinics regarding the presence of current glucocorticoid treatment and other study-entry criteria, 53 patients were randomised to receive metformin ( $\mathrm{n}=26$ subjects) or placebo $(\mathrm{n}=27)$ for 12 weeks (Figure 1). Participants were allocated to metformin or placebo treatments (in a 1:1 ratio) according to a pre-specified, computer-generated randomisation table, using blocks of four, stratified according to age ( $\leq 45$ years or $>45)$ and BMI $\left(\leq 27 \mathrm{~kg} / \mathrm{m}^{2}\right.$ or $\left.>27\right)$. Sequentially numbered containers with metformin or placebo tablets of identical appearance, size, weight, and taste were issued to the eligible patients directly by the Pharmacy. Participants, investigators and treating physicians were blinded to the treatment allocation.

Identical metformin (850mg; Merck Serono) or placebo tablets (850mg; Delpharm Laboratories) were administered in escalating doses to reduce gastrointestinal adverse events, aiming for $3 \times 850 \mathrm{mg} /$ day: $850 \mathrm{mg} /$ day for the first 5 days, $2 \times 850 \mathrm{mg} /$ day for the next 5 days and full-dose subsequently. Study drug was administered for 12 weeks. The glucocorticoids were titrated by the treating specialists, independently of the study, based on patients' clinical needs, with glucocorticoids dose reduced as soon as clinical condition would allow. Four 
patients withdrew due to gastrointestinal intolerance of the minimum acceptable dose $(2 \times 850 \mathrm{mg})$ in the metformin group. Two patients withdrew from the placebo group due to the development of overt diabetes mellitus, and one for the glucocorticoid dose falling outside of inclusion criteria. Three patients in each group discontinued due to inability to adhere to the appointment schedule. This left 19 subjects on metformin and 21 on placebo for analysis (Figure 1).

The study was approved by an independent Ethics Committee (South East Research Ethics Committee, REC09/H1102/82) and conducted according to the Declaration of Helsinki. All patients gave written informed consent.

\section{Assessments}

The primary outcome was the between-group change in the visceral-to-subcutaneous fat area ratio over 12 weeks, assessed by computed tomography $(\mathrm{CT})^{11}$, as this parameter, a recognised surrogate for metabolic risk $^{12}$, was found to be markedly raised in patients with Cushing's syndrome compared to matched subjects with simple obesity $^{11}$, with the cortisol burden corresponding to a reduced AMPK activity in visceral fat ${ }^{8}$ being reversedby metformin in vitro ${ }^{9}$. Secondary outcomes involved changes in the following parameters: anthropometric measurements, insulin resistance and $\beta$-cell function by the homeostasis model assessment (HOMA2) ${ }^{13}$, fasting and post $75 \mathrm{~g}$ sucrose challenge fibroblast growth factor 21 (FGF21), glucose, and tumour necrosis factor-alpha (TNF $\alpha$ ), lipid profile, high sensitivity C-reactive protein (hsCRP), fibrin clot properties, $\beta$-C-terminal telopeptide $(\beta \mathrm{CTX})$, procollagen type $1 \mathrm{~N}$-terminal propeptide (P1NP), and osteocalcin concentrations, bone density by dual-energy X-ray absorptiometry (DEXA), and carotid intima-media thickness (IMT) by Doppler ultrasound. Appetite was examined using visual analogue scales (VAS) and the 'Three factor eating questionnaire, ${ }^{14}$. Physical activity was assessed via VAS and the National Audit of Cardiac Rehabilitation Minimum Dataset Short Physical Activity Questionnaire ${ }^{15}$. Symptom severity/intrinsic disease activity were evaluated by relevant VAS, and safety and clinical impact by types and the occurrence of adverse events. 
Imaging and carbohydrate challenge were performed at 0 and 12 weeks. Physical examination, VAS and fasting blood sampling were conducted at 4-week intervals. Details of the imaging and assay protocols are described in the Supplementary Methods.

\section{Statistical analysis}

Sample size calculation for the primary outcome was based on differences between patients with endogenous Cushing's syndrome and matched subjects with simple obesity ${ }^{11}$. Considering that the difference in visceralto-subcutaneous adipose tissue area ratio was $0.845 \pm 0.525(\mathrm{n}=24)$ for patients with Cushing's syndrome and $0.28 \pm 0.17(\mathrm{n}=10)$ for obese controls, this results in a population mean difference of 0.57 with a standard deviation using pooled estimate of variance of $0.45 .90 \%$ power at $5 \%$ significance, the estimated sample sizes were 15 experimental subjects and 15 controls. Allowing for the short duration of treatment and heterogeneity of the study population with inflammation on exogenous glucocorticoids, the target was 20 patients completing the study in each arm. Our analysis followed the modified intention-to-treat principle in which individuals completing the study were analysed in the groups to which they were randomised (Supplementary Material). For continuous data, normality was assessed by the Shapiro-Wilk normality test and comparison of variances by the F-test; two-sample t-test for equal variances or Mann-Whitney tests were used for betweengroup analysis; paired t-test or Wilcoxon signed-rank test were conducted for within-group comparisons. Parametric data are reported as a mean \pm standard deviation, non-parametric as a median and interquartile range (IQR). Group analysis was performed by repeated-measures two-way ANOVA. Multivariate regression model was used for adjustment for baseline variables as required. Mixed-effect regression was done for assessment of covariants at different time-points. Categorical data were examined by chi-square or Fisher's exact tests in cases of few events. Pre-determined relevant correlations were computed by Pearson or Spearman rank-tests (correlation strength was defined by r-value: 0.3 to 0.69 (moderate), $\geq 0.7$ (strong)). Given small group sizes, analysis reflects all available data without adjustment for missing data and the results were not adjusted for multiple testing. A p-value of $<0.05$ was considered statistically significant. 


\section{RESULTS}

\section{Baseline characteristics}

Patients in the two treatment groups, although heterogeneous, were well-balanced in terms of age, sex, BMI and underlying diagnosis both at randomisation (Table 1) and from those completing the study (Table 2, Table S1\&S3-12). There were no statistically significant differences in glucocorticoid exposure prior and during the study between the treatment groups (Table 1, Table S1). For patients completing the study (Table S1), the duration of continuous glucocorticoid treatment at recruitment was 36 (IQR 10-156) vs. 36 (IQR 7.5-126) months (metformin vs. placebo; $\mathrm{p}=0.67$ ) and the cumulative exposure over the study period was $1860 \mathrm{mg}$ prednisolone-equivalent (IQR 1060-2810) vs. 1770mg (IQR 1020-2356) (metformin vs. placebo; p=0.76), having received continuous glucocorticoid treatment.

There was no between-group difference for participants' baseline physical activity (Table 1, Table S1), which was very low. Although all participants received the same advice on benefits of healthy eating and physical activity at the start, only the placebo-treated patients reported a mild increase in physical activity over the course of the study (Figure S1). Metformin and placebo treated patients did not match at randomisation and throughout the study for one aspect of feeding behaviour, the cognitive restraint of eating (Table 1, Table S6), where placebo-treated patients scored significantly higher (suggestive they could more easily follow dietary restrictions). We have included these lifestyle factors, which in theory could favour the placebo-treated arm, in an adjusted model for our primary outcome. The treatment adherence was similar between patients taking metformin and the placebo, $88 \pm 3 \%$ vs. $88 \pm 2 \%$, metformin vs. placebo, $\mathrm{p}=0.85$.

\section{Main findings}

\section{Effect of metformin on body composition}


The primary outcome, visceral-to-subcutaneous ratio, did not change in metformin-treated patients (Table 2). The truncal subcutaneous fat area decreased in metformin-treated patients relative to placebo (Figure 2a, Table 2) over 12 weeks, but not visceral adiposity $\left(+2501 \pm 4891 \mathrm{~mm}^{2}\right.$ vs. $+443 \pm 6810$, metformin vs. placebo, $\left.\mathrm{p}=0.28\right)$. Adjustment for baseline variables (difference in self-reported exercise, attitude to diet and cumulative glucocorticoid exposure, ethnicity, BMI and FGF21) in a multivariate regression model did not alter the above conclusions (Table S2).

Changes in weight and waist circumference did not reach statistical significance (Table 2, Table S3). On physical examination, two (10\%) metformin-treated vs. $11(52 \%)$ placebo-treated patients were noted to have more pronounced characteristic facial adiposity associated with Cushing's syndrome, a so-called 'moon face'. $(\mathrm{p}=0.007)$.

\section{Effect of metformin on carbohydrate and lipid metabolism and the liver function}

Glucose and HbA1c decreased in metformin-treated subjects (Figure 2b, Table 2), reducing the number of patients with dysglycaemia (defined as fasting glucose $\geq 6.1 \mathrm{mmol} / \mathrm{L}$ or any $2 \mathrm{~h}$ post-challenge glucose $\geq 7.8^{16}$ ). At baseline, 4 patients in each group had dysglycaemia, whilst at the end of the study none of the metformintreated and $7(33 \%)$ of the placebo-treated patients were dysglycaemic $(\mathrm{p}=0.009)$. Metformin prevented worsening of insulin resistance (HOMA2IR) (Figure 2c, Table 2) and improved $\beta$-cell function, estimated as the HOMA2\%B/HOMA2IR disposition index (Figure 2d, Table 2), accounting for a compensatory increase in insulin secretion. The lipid profile (Figure 2e, Table 2, Table S4) and liver function tests (Figure 2f, Table S5) improved in metformin-treated subjects. Changes in AST/ALT $(r=-0.47, \mathrm{p}=0.02)$ and GGT $(\mathrm{r}=0.55, \mathrm{p}=0.008)$ correlated with insulin resistance. Considering metabolic syndrome risk factors, the worse were the initial parameters, the greater were the improvements in waist circumference $(\mathrm{r}=-0.57, \mathrm{p}=0.01)$, HOMA2IR $(\mathrm{r}=-0.54$, $\mathrm{p}=0.02)$, glucose $(\mathrm{r}=-0.62, \mathrm{p}=0.005)$, triglycerides $(\mathrm{r}=-0.63, \mathrm{p}=0.004)$, LDL $(\mathrm{r}=-0.46, \mathrm{p}=0.046)$ and HDL $(\mathrm{r}=-$ $0.49, \mathrm{p}=0.001$ ) with metformin over the study period. Metformin had no effect on blood pressure. 


\section{Effect of metformin on appetite}

Hunger (Figure 2g, Table S6), sugar craving (Figure $2 \mathrm{~h}$ ) and disinhibition of eating $(\mathrm{p}=0.02)$ (Table S6) were reduced in metformin-treated subjects. Glucocorticoid treatment doses correlated positively to hunger ( $\mathrm{r}=0.33$, $\mathrm{p}=0.04)$ and sugar craving $(\mathrm{r}=0.40, \mathrm{p}=0.02)$. The hunger scores, sugar craving and disinhibition correlated positively to fat body composition changes and insulin resistance (Supplementary Material).

\section{Effect of metformin on FGF21 and adiponectin}

Fasting FGF21 and adiponectin concentrations increased over 12 weeks in metformin-treated patients (Table 2). We identified an increase in FGF21 in response to sucrose challenge (76.2pg/mL (IQR 33.3-168.0) to 154.8 (IQR 50.8-472.6), baseline to peak, $\mathrm{n}=50, \mathrm{p}=0.003$ ), but there was no between-group post-challenge difference (Table 2). FGF21 and adiponectin changes correlated significantly with nearly all favourable study outcomes (Supplementary Material).

\section{Effect of metformin on fibrinolysis and intima-media thickness}

Fibrin clot lysis time (Figure 3a, Table 2), an assay measuring fibrinolytic potential, was reduced in metformintreated subjects while there was no significant effect on maximum absorbance, a parameter assessing clot density and fibre thickness (Table S7). Progression of IMT was attenuated in metformin-treated subjects (Table 2). The higher the entry IMT, the greater was any IMT reduction on metformin $(r=-0.46, p=0.048)$.

\section{Effect of metformin on bone turnover and bone mass}


The bone resorption marker $\beta \mathrm{CTX}$ decreased in metformin-treated patients (Figure 3e-f). P1NP, representing bone formation, and osteocalcin, reflecting both formation and resorption, did not change significantly (Table S8-S12). The treatment groups were matched for bisphosphonate treatment and vitamin D concentrations (Table S12). Bone mineral density (BMD) at the hip (Figure 3d, Table 2\&S8-S9) increased in metformintreated patients. This increase persisted after adjustment for changes in hip circumference and weight in a multivariate regression model $(\mathrm{p}=0.02)$. Three $(19 \%)$ metformin-treated subjects reduced their hip BMD compared to $14(67 \%)$ patients on placebo $(\mathrm{p}=0.007)$. No changes were identified at the spine (Table S8-S9).

\section{Effect of metformin on inflammation and clinical safety outcomes}

hsCRP decreased in metformin-treated subjects compared to placebo (Figure 3b, Table 2). Total white cellcount and neutrophil count reduced from baseline within the metformin group but the between-group

difference did not reach statistical significance (Table 2). Carbohydrate-challenged TNF $\alpha$ levels increased significantly compared to pre-treatment in the placebo cohort $(p=0.04)$, but not in the metformin group (Figure S2). In a mixed effect regression model incorporating glucocorticoid dose change during the study, white cellcount decreased with decreasing glucocorticoid dose by $0.07 \times 10^{9} / \mathrm{L} / \mathrm{mg}$ prednisolone-equivalent decrease $(p<0.001)$, with no statistically significant difference between metformin and placebo. Neutrophils decreased with decreasing glucocorticoid dose by $0.06 \times 10^{9} / \mathrm{L} / \mathrm{mg}$ prednisolone-equivalent decrease $(\mathrm{p}=0.003)$ with no statistically significant difference between metformin and placebo (difference $0.49 ; \mathrm{p}=0.60$ ). hsCRP did not change with decreasing glucocorticoid dose (0.00006 increase per unit decrease in glucocorticoid dose; $\mathrm{p}=0.69$ ) and there was a statistically significant difference between metformin and placebo (difference -0.1 ; $\mathrm{p}=0.03)$.

Metformin-treated patients with respiratory conditions reported reduced dyspnoea (Figure 3c). Patients with rheumatic diseases taking metformin improved their global disease activity score (Table 2). There were more episodes of pneumonia, more severe infections and more respiratory and overall serious adverse events in the placebo arm (Table 3). Diarrhoea occurred more frequently in metformin-treated subjects (Table 3). 


\section{DISCUSSION}

In this study, metformin administration showed superiority to placebo in improving the metabolic profile and clinical outcomes in patients without known diabetes established on systemic glucocorticoid treatment for a chronic inflammatory disease.

While no change was observed for the visceral-to-subcutaneous fat ratio (primary outcome) or the visceral fat mass during the 3-months study period, a modest reduction in truncal subcutaneous fat was seen in the metformin-treated patients. Metformin also appeared to prevent progression of facial adiposity. Truncal subcutaneous fat is known to expand in hypercortisolaemia ${ }^{17}$, alongside visceral fat ${ }^{11,17}$, and higher truncal subcutaneous fat contributes to the adverse cardiometabolic risk profile associated with central adiposity ${ }^{18}$. The visceral to subcutaneous ratio has been associated with cardiometabolic risk above and beyond body mass index and visceral adiposity in the Framingham Heart Study ${ }^{12}$. Metformin has been shown to qualitatively improve visceral fat irrespective of fat mass $^{19}$ and to reduce abdominal subcutaneous fat in overweight patients with type 2 diabetes (T2DM) ${ }^{20}$, a cohort with the best cardiovascular outcome in the United Kingdom Prospective Diabetes Study ${ }^{7,21}$. Whilst glucocorticoid effects on adipose functionality are insufficiently known, our data suggest a multisystem metabolic advantage with glucocorticoid-metformin co-administration. The AMPK-pathway may represent one of the mediators of glucocorticoid-metformin interaction ${ }^{8,9}$. Metformin may interact with glucocorticoid metabolism via $11 \beta$-hydroxysteroid dehydrogenase- $1^{22}$, although this may not play a role in patients with supraphysiological doses of synthetic glucocorticoid treatment.

Insulin resistance, $\beta$-cell function and glucose levels were improved in metformin-treated subjects compared to placebo which is clinically relevant, given the cohort's propensity to diabetes. These findings together with improved liver function are in keeping with the reduced conversion from pre-diabetes to T2DM and improved liver function in metformin-treated subjects in the Diabetes Prevention Program ${ }^{23,24}$. Glucocorticoids increase glucose levels both at non-diabetic as well as diabetic glucose ranges ${ }^{25}$. Metformin's ability to antagonise this glucose rise is important, as cardiovascular events increase linearly with rising glucose even in the non-diabetic 
glucose range ${ }^{26}$. Moreover, increasing glucose often associates with poor outcomes ${ }^{16}$ and metformin has been shown to improve clinical outcomes where glucose parameters decreased in non-diabetic patients ${ }^{7,21}$.

Glucocorticoid therapy, which can paradoxically induce a pro-inflammatory phenotype overlapping with the metabolic syndrome, is associated with increased cardiovascular morbidity and mortality ${ }^{5,6}$. The more florid the initial metabolic disturbances in our patients, the greater the detected benefits of metformin. Our finding of $19 \%$ lower LDL in statin-naïve metformin-treated patients, in line with reports from pre-diabetic cohorts ${ }^{7}$, may represent a considerable clinical benefit. Every $1 \%$ reduction in LDL by statins was associated with up to $2.5 \%$ lower cardiovascular events in inflammatory disease where glucocorticoid-related lipid side-effects can be confounded by their anti-inflammatory action ${ }^{27}$.

Appetite stimulation by glucocorticoids represents a major drive for obesity ${ }^{28}$. Here we show that metformin, a drug with anorectic effects ${ }^{7}$, was associated with suppressed appetite and sugar craving, promoting abdominal fat loss, even when co-administered with glucocorticoids. Supporting this, glucocorticoids increased appetite in rodents, recruiting AMPK, an effect mitigated by metformin in vitro ${ }^{9}$.

Fructose overconsumption, associated with adverse metabolic effects and altered glucocorticoid signalling, could contribute to a Cushingoid phenotype ${ }^{29}$. FGF21, a biomarker of fructose metabolism and crucial in cardiometabolic protection, robustly and rapidly increases in response to fructose ingestion differentially from glucose $^{29}$. Here we identified a rise in FGF21 after a sucrose challenge in all groups. Metformin prevented a drop in fasting FGF21 and increased adiponectin, with the changes correlating favourably with nearly all study outcomes. We hypothesise that metformin may reduce glucocorticoid-related metabolic dysfunction and systemic inflammation by enhancing the FGF21-adiponectin axis.

Both poorly-treated inflammatory disease and glucocorticoid overexposure represent prothrombotic phenotypes, associated with impaired fibrinolysis and an accelerated atherosclerosis ${ }^{6,30}$. Prolonged fibrin clot lysis time is an independent predictor of cardiovascular mortality ${ }^{31}$; here we demonstrate enhanced fibrinolysis in metformintreated patients. Metformin is believed to improve the hypofibrinolytic environment in T2DM, with this effect 
proposed to contribute substantially to the reduced cardiovascular risk attributed to metformin in the United Kingdom Prospective Diabetes Study ${ }^{32}$. Our data mark potentially a major advantage for metformin in reducing thrombosis risk in glucocorticoid-treated patients.

IMT is a widely accepted surrogate marker for a generalised clinical/subclinical atherosclerosis, correlating to the risk of development of atherosclerotic plaques and coronary events in the majority of trials ${ }^{33}$. Metformin treatment was associated with a reduced progression of IMT in this heterogeneous cohort, similar to the benefits identified in subjects with the metabolic syndrome of $\mathrm{T} 1 \mathrm{DM} \& \mathrm{~T} 2 \mathrm{DM}^{7,33,34}$ unlike in non-diabetic patients established on statins for coronary disease where metformin did not reduce $\mathrm{LDL}^{35}$. A longer treatment study is warranted to probe into the mechanistic pathways that are responsible for the protective effects of metformin in patients on glucocorticoids.

There was a reduction in inflammation (hsCRP) and improvement in patient-oriented clinical markers of disease activity in metformin-treated patients who also appeared to have a better infection profile. This is clinically relevant, given the participants' severe immunosuppression. There were fewer episodes of pneumonia, milder infective events, no severe asthma exacerbation and less overall serious adverse events in metformin-treated patients. Although warranting an exploration in a larger study, these results point to up to $30 \%$ absolute risk reduction of moderate-to-severe infections or all-cause hospital admissions, which corresponds to treating 3 patients with metformin to prevent one case. The beneficial effect of lower glucose levels on immunomodulation is likely. Nonetheless, there is growing evidence that metformin has discrete anti-inflammatory properties beyond this ${ }^{36}$. In experimental models, metformin prevented lung infections independently of glycaemia and reduced the inflammation of chronic asthma, uveitis and rheumatoid arthritis $7,37,38$. Metformin appeared to be superior to other glucose-lowering agents at reducing infections in $\mathrm{T}_{2} \mathrm{DM}^{38,39}$ and compared to placebo in $\mathrm{PCOS}^{40}$, independently of glycaemia. Moreover, others linked a greater burden of adverse events with a higher disease activity and reduced remissions ${ }^{41}$. As an acute treatment for chronic obstructive pulmonary disease exacerbations, metformin did not benefit non-diabetic individuals in a very short trial ${ }^{37}$, while in the chronic setting prescribing metformin in T2DM was associated with lower hospitalisation in patients with asthma or 
chronic obstructive pulmonary disease ${ }^{37}$. Glucocorticoid effects on the immune system are complex, and metformin's interactions would deserve further exploration with immune profiling in a dedicated study ${ }^{42}$.

Glucocorticoids are renowned for adverse bone effects ${ }^{6}$. In our metformin-treated patients there was a modest reduction in the bone resorption marker $\beta \mathrm{CTX}$, with the overall bone turnover consistent with a bone mass increase, and a subtle bone mass increase was identified at the hip. This effect appeared to persist irrespective of the body composition assessed, vitamin D levels or bisphosphonate treatment, warranting further studies with greater patient numbers and using other assessment modalities to characterise the effect of confounding factors in more detail, and to explore the clinical significance of these signal findings. Metformin prevented glucocorticoid-induced bone loss in the femur of an animal model, differentially to the mode of action of alendronate ${ }^{43}$. Advantageous effects of metformin on fracture risk or bone mass were noted in subjects with $\mathrm{T}_{2} \mathrm{DM}^{44}$ and in $\mathrm{PCOS}^{40}$, possibly by modulating metabolic-immune interplay ${ }^{7}$.

Glucocorticoid-related metabolic dysfunction offsets the drug's profound anti-inflammatory benefits, contributing to the lasting increased morbidity associated with inflammatory diseases ${ }^{5,6}$. Metformin is effective in treating the endothelial dysfunction and the metabolic syndrome of $\operatorname{PCOS}^{7,45}$ and preventing glycaemic deterioration when initiated simulataneously with glucocorticoids in non-diabetic individuals ${ }^{10}$. Here we show wider metabolic and clinical benefits in subjects already established on glucocorticoid treatment. While metformin has shown favourable effects in different insulin resistant pathological states, no other studies have been conducted to explore the role of this agent on vascular, thrombotic, inflammatory and bone markers following glucocorticoid treatment. The morbidity of the underlying disease and the glucocorticoid-driven increased appetite often limit intervention by lifestyle change. We suggest that metformin used in glucocorticoid-treated patients without diabetes may have the potential for improving treatment-related complications and cardiovascular prognosis.

Many patients on glucocorticoid tretament develop diabetes. Our recent study, for example, showed worsening metabolic parameters (basal and 2h AUC glucose and cholesterol) in glucocortioid-treated patients (previously not on glucocorticoids) on placebo compared to metformin treatment ${ }^{10}$. Our current study population on chronic 
glucococortcoid treatment, who did not have overt diabetes at study entry might, therefore, be somewhat less predisposed to diabetes. We did not expect in our placebo group that metabolic parameters would worsen during the 12-week study period, especially in face of reducing glucocorticoid doses over the 12-weeks (Table 1).

Indeed, only a few parameters showed modest worsening in the placebo group (Table 1 and Supplementary Material). However, our data showed significant improvement in various parameters in the metformin-treated group. These results raise the intriguing possibility to treat chronic inflammatory disease patients with metformin even without concomitant glucocorticoid medication.

\section{Strength and weaknesses}

The strength of our study is its randomised, double-blind design in largely well-balanced treatment cohorts. Selected patients typify subjects with chronic active inflammation at a high risk for metabolic complications of iatrogenic Cushing's syndrome not offered metformin in real-life practice. Our results are clinically pertinent and the cohort heterogeneity adds to the data generalizability. Metformin is available worldwide and could improve metabolic status of large number of patients; according to a recent study $3 \%$ of the overall general population, with even higher percentages in the older population ( $7 \%$ of $60-79$ and $10 \%$ of over 80 years old subjects $)^{2}$.

Our study has several limitations, the main ones being the small sample size, heterogeneity of patients and the relatively short treatment duration. The initial sample size calculation was derived from records on patients with endogenous Cushing's syndrome ${ }^{11}$, although data in patients with rheumatoid arthritis and other conditions supported our calculations ${ }^{46-48}$. While various biases may affect parameters at different time points impacting on the final conclusions, the direction of changes favouring metformin in the assessments appears consistent. Given the small sample size, we report unadjusted findings; selection bias due our drop-out patients cannot be fully excluded, although the remaining groups remained well-balanced and the drop-out rate was similar in both groups. The cohort size, heterogeneity and treatment duration limit the scope for exploring disease-specific and long-term clinical outcomes of interest. Given the sample size and relatively large number of secondary 
outcomes, cautious interpretation is warranted; nevertheless, our data are concordant and contributory to the growing evidence of metformin's benefits involving robust numbers of patients, especially those at diabetes risk. A larger study may help to both identify an optimal cohort of patients who would benefit most from this intervention and explore the subsequent clinical benefits suggested by this trial.

In summary: We have carried out a randomised, double-blind, placebo-controlled, phase 2 proof-of-concept trial exploring the potential of metformin to improve the metabolic profile of established glucocorticoid treatment in patients without known diabetes burdened with chronic active inflammation. We have shown that metformin-treated patients showed a significant improvement in several prognostic parameters and clinical outcomes. Compared to placebo, metformin-treated participants had reduced appetite, truncal and facial subcutaneous fat and insulin resistance, improved $\beta$-cell function, glycaemia, lipid profile, liver function, fibrinolysis, subclinical atherosclerosis, bone metabolism, infection risk, inflammation, disease activity and symptom severity, as well as fewer hospital admissions. These results may indicate the possibilty of a major improvement in patient care and justify further research into the concomitant use of metformin in patients on glucocorticoid treatment. 


\section{Acknowledgement}

We would like to thank all the patients who participated in the study and the physicians, nurses, clinical scientists and collaborators who enabled us to conduct the study. Special thanks to Professor Neil Barnes for logistic support.

The study was supported by a Project Grant from Barts Charity. We are grateful for Merck Serono for providing the study drug and the matching placebo, and for financial support. Study design, data collection, statistical analysis and data interpretation were performed independently of the study funders.

Registration: ClinicalTrials.gov number NCT01319994

\section{Data sharing}

Study protocol and individual participant data that underlie the results reported in this article will be available after de-identification (text, tables, figures, and appendices) 9-36 months after publication to researchers who have signed data access agreement and provide a methodologically sound proposal for individual participant data meta-analysis. Proposals should be directed to the corresponding author. 


\section{Table 1: Baseline characteristics}

Reported as a mean \pm standard deviation or as median (IQR: interquartile range) or as number (\% out of randomized patients)

\begin{tabular}{|c|c|c|c|}
\hline & Metformin & Placebo & $\begin{array}{l}\text { P- } \\
\text { value }\end{array}$ \\
\hline Allocated number of patients & 26 & 27 & 0.99 \\
\hline Age & $47 \pm 15$ & $45 \pm 15$ & 0.63 \\
\hline Sex-female (\%) & $14(54 \%)$ & $15(56 \%)$ & 0.99 \\
\hline Ethnicity & Mixed & Mixed & 0.31 \\
\hline - White & $12(46 \%)$ & $17(63 \%)$ & \\
\hline - South Asian & $6(23 \%)$ & $5(18 \%)$ & \\
\hline - Black/African American & $5(19 \%)$ & $5(18 \%)$ & \\
\hline - Other (oriental; mixed White/South Asian) & $3(12 \%)$ & 0 & \\
\hline$B M I\left(\mathrm{~kg} / \mathrm{m}^{2}\right)$ & 27.3 (IQR 23.3 to 36.1 ) & 28.5 (IQR 24.7 to 35.8 ) & 0.64 \\
\hline Current smoker & $6(23 \%)$ & $2(7 \%)$ & 0.14 \\
\hline Smoking pack history & 0 (IQR 0 to 6.3 ) & 0 (IQR 0 to 5.0$)$ & 0.94 \\
\hline Main indication for GC treatment & Mixed & Mixed & 0.21 \\
\hline - Asthma & $7(27 \%)$ & $9(33 \%)$ & \\
\hline - Vasculitis (GCA/PNA/Wegener's, non-specified) & $7(27 \%)$ & $3(11 \%)$ & \\
\hline - Sarcoidosis & $5(19 \%)$ & $1(4 \%)$ & \\
\hline$-S L E$ & $4(15 \%)$ & $5(19 \%)$ & \\
\hline$-R A$ & $1(4 \%)$ & $2(7 \%)$ & \\
\hline - Interstitial lung disease (other than connective tissue disease-related) & $1(4 \%)$ & $2(7 \%)$ & \\
\hline - Other (mixed connective tissue disease; isolated uveitis/scleritis/retinitis) & $1(4 \%)$ & $1(4 \%)$ & \\
\hline - Myositis (other than SLE-related) & 0 & $4(15 \%)$ & \\
\hline Duration of continuous systemic glucocorticoid treatment by the study entry (months) & 24 (IQR 6 to 129$)$ & $36(\mathrm{IQR} 5$ to 120$)$ & 0.98 \\
\hline Estimated cumulative dose of glucocorticoids by the study entry (equivalent mg of prednisolone) & 16643 (IQR 2643 to 103275 ) & 20860 (IQR 8360 to 64400 ) & 0.92 \\
\hline Cumulative dose of glucocorticoid last 3 months prior to the study entry (equivalent mg prednisolone) & 1845 (IQR 1273 to 2700$)$ & 2010 (IQR 1295 to 2930 ) & 0.45 \\
\hline Cumulative dose of glucocorticoids during the study (equivalent mg of prednisolone) & $1782 \pm 1092$ & $1718 \pm 805$ & 0.82 \\
\hline Prednisolone equivalent dose (mg) at the initial study visit & 23 (IQR 12 to 40$)$ & 25 (IQR 12 to 40$)$ & 0.83 \\
\hline Prednisolone-equivalent dose (mg) at 4 weeks & 20 (IQR 10 to 28$)$ & 20 (IQR 10 to 29$)$ & 0.98 \\
\hline Prednisolone-equivalent dose (mg) at 8 weeks & 20 (IQR 10 to 30$)$ & $18(10$ to 22$)$ & 0.53 \\
\hline Prednisolone-equivalent dose (mg) at 12 weeks & $13(\mathrm{IQR} 10-25)$ & 15 (IQR 10-25) & 0.62 \\
\hline Waist circumference $(\mathrm{cm})$ & $96.9 \pm 17.3$ & $100.2 \pm 19.4$ & 0.52 \\
\hline Hip circumference (cm) & $106.5 \pm 16.1$ & $108.9 \pm 14.2$ & 0.48 \\
\hline Systolic BP $(\mathrm{mm} \mathrm{Hg})$ & $121 \pm 16$ & $129 \pm 20$ & 0.14 \\
\hline
\end{tabular}




\begin{tabular}{|c|c|c|c|}
\hline & Metformin & Placebo & $\begin{array}{l}\text { P- } \\
\text { value }\end{array}$ \\
\hline Diastolic BP (mm Hg) & $79 \pm 12$ & $83 \pm 12$ & 0.26 \\
\hline Visceral abdominal fat (mm2) & 22646 (IQR 16378 to 30746$)$ & 25984 (IQR 17211 to 35355$)$ & 0.36 \\
\hline Subcutaneous abdominal fat ( $\mathrm{mm} 2$ ) & 48715 (IQR 36614 to 65124 ) & 48485 (IQR 33278 to 62939 ) & 0.98 \\
\hline Visceral-to-subcutaneous fat ratio & 0.44 (IQR 0.33 to 0.67 ) & 0.58 (IQR 0.34 to 0.84 ) & 0.49 \\
\hline Fasting glucose $(\mathrm{mmol} / \mathrm{L})$ & 4.7 (IQR 4.5 to 5.7$)$ & 4.9 (IQR 4.5 to 5.8 ) & 0.38 \\
\hline AUC glucose (mmol/L*min) post $75 \mathrm{~g}$ sucrose challenge & 826 (IQR 643 to 1054 ) & 806 (IQR 717 to 1179 ) & 0.87 \\
\hline $\mathrm{HbAlc}(\mathrm{mmol} / \mathrm{mol})$ & $40 \pm 6$ & $39 \pm 7$ & 0.69 \\
\hline HOMA2IR & 4.7 (IQR 2.2 to 5.5 ) & 4.1 (IQR 2.8 to 5.4$)$ & 0.97 \\
\hline$H O M A 2 \% B$ & $282.8 \pm 132.9$ & $254.9 \pm 124.6$ & 0.43 \\
\hline$H O M A 2 \% B / H O M A 2 I R$ & $68.3 \pm 21.0$ & $63.8 \pm 24.8$ & 0.48 \\
\hline Total cholesterol (mmol/L) & 5.6 (IQR 4.7 to 6.4$)$ & 5.4 (IQR 4.7 to 6.2 ) & 0.66 \\
\hline$L D L(\mathrm{mmol} / \mathrm{L})$ & 2.8 (IQR 2.3 to 3.4 ) & 2.9 (IQR 2.5 to 3.4 ) & 0.71 \\
\hline$H D L(\mathrm{mmol} / \mathrm{L})$ & $1.86 \pm 0.52$ & $1.81 \pm 0.46$ & 0.67 \\
\hline Triglycerides $(\mathrm{mmol} / \mathrm{L})$ & 1.5 (IQR 1.0 to 2.3 ) & 1.3 (IQR 1.1 to 1.8 ) & 0.29 \\
\hline$A L T(U / L)$ & 17 (IQR 14 to 25$)$ & 19 (IQR 14 to 26$)$ & 0.53 \\
\hline$A L P(U / L)$ & $62 \pm 15$ & $59 \pm 19$ & 0.55 \\
\hline$F G F 21(\mathrm{pg} / \mathrm{mL})$ & 56.5 (IQR 27.3 to 156.3 ) & 96.0 (IQR 41.5 to 256.8 ) & 0.25 \\
\hline Adiponectin $(n g / m L)$ & 22.0 (IQR 11.6 to 44.9 ) & 21.8 (IQR 8.6 to 41.5 ) & 0.60 \\
\hline$h_{s} C R P(m g / L)$ & 0.033 (IQR 0.009 to 0.049 ) & 0.025 (IQR 0.010 to 0.050 ) & 0.96 \\
\hline Haemoglobin & $13.2 \pm 0.3$ & $13.1 \pm 0.3$ & 0.89 \\
\hline White cell count & $10.2 \pm 0.7$ & $8.7 \pm 0.8$ & 0.18 \\
\hline Neutrophils & 7.4 (IQR 5.2 to 8.7 ) & 5.8 (IQR 3.5 to 8.5 ) & 0.15 \\
\hline Lymphocytes & 1.7 (IQR 1.2 to 2.8 ) & 1.3 (IQR 0.8 to 2 ) & 0.08 \\
\hline Monocytes & $0.6 \pm 0.1$ & $0.6 \pm 0.1$ & 0.91 \\
\hline Eosinophils & 0.1 (IQR 0.1 to 0.2 ) & 0.1 (IQR 0 to 0.2 ) & 0.23 \\
\hline Basophils & $0(\mathrm{IQR} 0$ to 0$)$ & 0 (IQR 0 to 0.03$)$ & 0.40 \\
\hline Platelets & 254 (IQR 203 to 327) & 233 (IQR 185 to 280 ) & 0.46 \\
\hline Intima-media thickness (mm) & 0.50 (IQR 0.46 to 0.55 ) & 0.51 (IQR 0.46 to 0.56 ) & 0.77 \\
\hline Lysis time (s) & 354 (IQR 276 to 426) & 366 (IQR 297 to 444) & 0.53 \\
\hline Lysis area $(A U)$ & 92.0 (IQR 60.7 to 126.7 ) & 120.7 (IQR 72.9 to 218.5 ) & 0.15 \\
\hline Total hip BMD (g/cm2) & $0.965 \pm 0.145$ & $0.988 \pm 0.195$ & 0.63 \\
\hline Spine $B M D(\mathrm{~g} / \mathrm{cm} 2)$ & $0.981 \pm 0.150$ & $0.997 \pm 0.168$ & 0.73 \\
\hline Vitamin $D(\mathrm{nmol} / \mathrm{L})$ & 45.5.0 (IQR 29.0 to 58.3) & 32.0 (IQR 25.0 to 71.5 ) & 0.62 \\
\hline$\beta C T X(\mu g / L)$ & 0.21 (IQR 0.17 to 0.33$)$ & 0.17 (IQR 0.08 to 0.31 ) & 0.35 \\
\hline$P 1 N P(\mu g / L)$ & 22.3 (IQR 13.9 to 34.7 ) & 20.5 (IQR 12.9 to 39.1 ) & 0.79 \\
\hline Osteocalcin $(\mu \mathrm{g} / L)$ & 9.3 (IQR 6.7 to 16.3 ) & 9.6 (IQR 5.2 to 14.6 ) & 0.64 \\
\hline
\end{tabular}

Page 20 of 37 


\begin{tabular}{|c|c|c|c|}
\hline & Metformin & Placebo & $\begin{array}{l}\text { P- } \\
\text { value }\end{array}$ \\
\hline Physical activity on the day of assessment (virtual scale 0-10.10 is the highest) & 2 (IQR 1 to 3.3 ) & 2 (IQR 1 to 3$)$ & 0.20 \\
\hline $\begin{array}{l}\text { Reported frequency of at least } 15 \text { min. of strenuous physical activity in } 7 \text { days (National Audit of } \\
\text { Cardiac Rehabilitation (NACR) Minimum Dataset Short Physical Activity questionnaire) }\end{array}$ & 0 (IQR 0 to 0$)$ & 0 (IQR 0 to 0$)$ & 0.28 \\
\hline Reported frequency of at least 15 min of moderate physical activity in 7 days (NACR questionnaire) & 0 (IQR 0 to 0.5$)$ & 0 (IQR 0 to 2$)$ & 0.84 \\
\hline $\begin{array}{l}\text { Reported frequency of at least } 15 \text { min of minimal effort physical activity in } 7 \text { days (NACR } \\
\text { questionnaire) }\end{array}$ & 3.5 (IQR 0 to 7$)$ & 3 (IQR 2 to 7 ) & 0.95 \\
\hline Cognitive restraint of eating $\wedge$ & $6 \pm 4$ & $9 \pm 4$ & 0.007 \\
\hline Disinhibition of eating ${ }^{\wedge}$ & 5 (IQR 3 to 10$)$ & 5 (IQR 2 to 8$)$ & 0.77 \\
\hline Hunger $\wedge$ & 4 (IQR 2 to 9) & 4. (IQR 2 to 8 ) & 0.99 \\
\hline \multicolumn{4}{|l|}{ Medications } \\
\hline Prednisolone & $25(96 \%)$ & $27(100 \%)$ & 0.49 \\
\hline Methylprednisolone & 0 & $1(4 \%)$ & 0.99 \\
\hline Beclomethasone & $1(4 \%)$ & $1(4 \%)$ & 0.99 \\
\hline Fluticasone & $4(15 \%)$ & $5(19 \%)$ & 0.99 \\
\hline Dexamethasone & $2(8 \%)$ & 0 & 0.22 \\
\hline Clobetasone & $1(4 \%)$ & 0 & 0.99 \\
\hline Methotrexate & $2(8 \%)$ & $3(11 \%)$ & 0.99 \\
\hline Hydroxychloroquine & $2(8 \%)$ & $4(15 \%)$ & 0.67 \\
\hline Azathioprine & $1(4 \%)$ & $1(4 \%)$ & 0.99 \\
\hline Mycophenolate & $1(4 \%)$ & $4(15 \%)$ & 0.35 \\
\hline Sulphasalazine & $1(4 \%)$ & 0 & 0.49 \\
\hline Ustekinumab & 0 & $1(4 \%)$ & 0.99 \\
\hline Tocilizumab & 0 & $1(4 \%)$ & 0.99 \\
\hline Omaluzimab & $1(4 \%)$ & $1(4 \%)$ & 0.99 \\
\hline Cyclophosphamide & 0 & $2(7 \%)$ & 0.49 \\
\hline Ciclosporin & $1(4 \%)$ & $1(4 \%)$ & 0.99 \\
\hline Bronchodilators & $11(42 \%)$ & $11(41 \%)$ & 0.99 \\
\hline Bisphosphonates & $12(46 \%)$ & $9(33 \%)$ & 0.41 \\
\hline $\mathrm{HRT} / \mathrm{OCP}$ & $3(12 \%)$ & 0 & 0.11 \\
\hline TB treatment & $2(8 \%)$ & $1(4 \%)$ & 0.61 \\
\hline Warfarin/LMWH & $1(4 \%)$ & $2(7 \%)$ & 0.99 \\
\hline Aspirin/clopidogrel & $3(12 \%)$ & $5(19 \%)$ & 0.70 \\
\hline Statins & $4(15 \%)$ & $1(4 \%)$ & 0.19 \\
\hline Antihypertensives & $12(46 \%)$ & $11(41 \%)$ & 0.79 \\
\hline Beta-blockers & $3(12 \%)$ & $1(4 \%)$ & 0.61 \\
\hline ACE-inhibitors/Sartans & $9(35 \%)$ & $8(30 \%)$ & 0.77 \\
\hline
\end{tabular}

Page 21 of 37 
MF, metformin; PL, placebo; BMI, body mass index; GC, glucocorticoid(s); GCA, giant cell arteritis; PNA, polyarteritis nodosa; SLE, systemic lupus erythematosus; RA, rheumatoid arthritis; LDL, lowdensity lipoprotein cholesterol; HDL, high-density lipoprotein cholesterol; ALT, alanine aminotransferase; ALP, alkaline phosphatase; FGF21, fibroblast growth factor 21; BCTX, B-C-terminal telopeptide; $\mathrm{P} 1 \mathrm{NP}$, procollagen type $1 \mathrm{~N}$-terminal propeptide; HRT, hormone replacement therapy; OCT, oral contraceptive therapy; LMWH, low molecular weight heparin; BMD, bone mineral density; AU, arbitrary units; ${ }^{\wedge}$ Components of the Three factor eating questionnaire

Page 22 of 37 


\section{Table 2. Outcome data}

Expressed as mean \pm standard deviation or median and interquartile range (IQR)

\begin{tabular}{|c|c|c|c|}
\hline & Metformin (MF) & Placebo (PL) & Difference between groups (P-value) \\
\hline \multicolumn{4}{|c|}{ Visceral-to-subcutaneous fat area (ratio) } \\
\hline Baseline (MF $n=19 ;$ PL $n=21)$ & 0.45 (IQR 0.32 to 0.77 ) & 0.60 (IQR 0.36 to 0.89 ) & 0.34 \\
\hline At 12 weeks & 0.56 (IQR 0.33 to 0.79 ) & 0.59 (IQR 0.37 to 0.81 ) & 0.92 \\
\hline Change over 12 weeks & $0.08 \pm 0.19$ & $-0.03 \pm 0.22$ & 0.09 \\
\hline Difference within groups (P-value) & 0.11 & 0.57 & \\
\hline \multicolumn{4}{|l|}{ Truncal subcutaneous fat area $\left(\mathrm{mm}^{2}\right)$} \\
\hline Baseline (MF $n=19 ;$ PL $n=21)$ & 48640 (IQR 33786 to 73959 ) & 41753 (IQR 32760 to 58873 ) & 0.70 \\
\hline At 12 weeks & 42643 (IQR 30519 to 72160 ) & 45214 (IQR 34394 to 57371 ) & 0.61 \\
\hline Change over 12 weeks & $-2035 \pm 4283$ & $1799 \pm 4859$ & 0.01 \\
\hline Difference within groups (P-value) & 0.053 & 0.12 & \\
\hline \multicolumn{4}{|l|}{ Weight (kg) } \\
\hline Baseline (MF $n=19, P L n=21)$ & 75(IQR 59.4 to 99) & 79 (IQR 68 to 97) & 0.56 \\
\hline At 12 weeks & 71 (IQR 58 to 103 ) & 80 (IQR 69 to 99 ) & 0.42 \\
\hline Change over 12 weeks & $-1.1 \pm 0.8$ & $0.5 \pm 0.5$ & 0.09 \\
\hline Difference within groups (p-value) & 0.11 & 0.47 & \\
\hline \multicolumn{4}{|l|}{ Fasting glucose $(\mathrm{mmol} / \mathrm{L})$} \\
\hline Baseline (MF $n=19 ;$ PL $n=21)$ & 5.0 (IQR 4.5 to 5.9 ) & 4.9 (IQR 4.5 to 5.4 ) & 0.82 \\
\hline At 12 weeks & 4.3 (IQR 4.1 to 4.6 ) & 5.0 (IQR 4.4 to 5.6$)$ & 0.03 \\
\hline Change over 12 weeks & -0.5 (IQR -0.8 to -0.3$)$ & 0 (IQR -0.4 to 0.3$)$ & 0.005 \\
\hline Difference within groups (P-value) & 0.001 & 0.89 & \\
\hline \multicolumn{4}{|c|}{ AUC glucose $\left(\mathrm{mmol} / \mathrm{L}^{*} \mathrm{~min}\right)$ post $75 \mathrm{~g}$ sucrose challenge } \\
\hline Baseline (MF $n=19 ;$ PL $n=20$ ) & 837 (IQR 636 to 1140 ) & 806 (IQR 712 to 1077 ) & 0.80 \\
\hline At 12 weeks & 711 (IQR 552 to 816 ) & 821 (IQR 749 to 1097) & 0.01 \\
\hline Change over 12 weeks & $-121.5(\mathrm{IQR}-306$ to -24$)$ & 30.8 (IQR -60.4 to 108.4$)$ & 0.002 \\
\hline
\end{tabular}

Page 23 of 37 


\begin{tabular}{|c|c|c|c|}
\hline Difference within groups (P-value) & 0.003 & 0.23 & \\
\hline \multicolumn{4}{|l|}{ HOMA2IR (Mass Units) } \\
\hline Baseline (MF $n=19 ;$; $L n=21)$ & 4.63 (IQR 2.09 to 4.88 ) & 3.82 (IQR 2.48 to 4.99 ) & 0.95 \\
\hline At 12 weeks & $4.53 \pm 2.1$ & $6.20 \pm 2.6$ & 0.03 \\
\hline Change over 12 weeks & $0.22 \pm 3.3$ & $2.35 \pm 3.2$ & 0.04 \\
\hline Difference within groups (P-value) & 0.61 & 0.003 & \\
\hline \multicolumn{4}{|l|}{ Disposition index (HOMA2\%B/HOMA2IR) } \\
\hline Baseline (MF $n=19 ;$ PL $n=21$ ) & $66.5 \pm 21.3$ & $69.5 \pm 23.2$ & 0.68 \\
\hline At 12 weeks & $83.7 \pm 21.9$ & $63.2 \pm 25.5$ & 0.01 \\
\hline Change over 12 weeks & $17.1 \pm 22.6$ & $-6.3 \pm 20.6$ & 0.001 \\
\hline Difference within groups (P-value) & 0.004 & 0.18 & \\
\hline \multicolumn{4}{|l|}{ LDL cholesterol $(\mathrm{mmol} / \mathrm{L})$} \\
\hline Baseline (MF $n=19, P L n=20)$ & $2.6(\mathrm{IQR} 2.3$ to 3.3$)$ & 2.9 (IQR 2.6 to 3.3$)$ & 0.33 \\
\hline At 12 weeks & 2.3 (IQR 1.9 to 2.7 ) & 3.0 (IQR 2.4 to 3.8$)$ & 0.01 \\
\hline Change over 12 weeks & $-0.4(\mathrm{IQR}-0.9$ to 0.3$)$ & 0.2 (IQR -0.4 to 0.5$)$ & 0.08 \\
\hline Difference within groups (P-value) & 0.04 & 0.68 & \\
\hline Cholesterol:HDL ratio (Atherogenic index) change over 12 weeks (MF $n=19 ;$ PL $n=21$ ) & $-0.26 \pm 0.85$ & $0.27 \pm 0.71$ & 0.04 \\
\hline \multicolumn{4}{|l|}{$h s C R P(m g / L)$} \\
\hline Baseline (MF $n=19$, PL $n=21)$ & $0.031 \pm 0.025$ & $0.033 \pm 0.024$ & 0.86 \\
\hline At 12 weeks & 0.016 (IQR 0.006 to 0.029$)$ & $0.036(\mathrm{IQR} 0.021$ to 0.050$)$ & 0.02 \\
\hline Change over 12 weeks & $-0.010 \pm 0.025$ & $0.003 \pm 0.026$ & 0.09 \\
\hline Difference within groups (P-value) & 0.08 & 0.5731 & \\
\hline$\%$ change over 12 weeks & $-40.9(\mathrm{IQR}-82.9$ to 45.4$)$ & 8.5 (IQR -30.3 to 128.6$)$ & 0.09 \\
\hline AUC of 4 visits over 12 weeks & $0.08 \pm 0.01$ & $0.12 \pm 0.02$ & 0.02 \\
\hline \multicolumn{4}{|l|}{ White cell-count $\left(10^{9} / L\right)$} \\
\hline Baseline (MF $n=19, P L n=19)$ & $9.9 \pm 0.9$ & $8.9 \pm 0.9$ & 0.40 \\
\hline
\end{tabular}

Page 24 of 37 


\begin{tabular}{|c|c|c|c|}
\hline At 12 weeks & 8.4 (IQR 6.6 to 10.3 ) & 7.4 (IQR 5.9 to 10.9 ) & 0.95 \\
\hline Change over 12 weeks & $-1.0($ IQR -2.4 to -0.1$)$ & -0.6 (IQR -2.0 to 0.8 ) & 0.25 \\
\hline Difference within groups (P-value) & 0.01 & 0.15 & \\
\hline \multicolumn{4}{|l|}{ Neutrophils } \\
\hline Baseline (MF $n=16 ; P L=17$ ) & 7.4 (IQR 4.5 to 9.6 ) & 5.8 (IQR 3.8 to 8.1 ) & 0.33 \\
\hline At 12 weeks & 4.6 (IQR 4 to 6.4 ) & 4.2 (IQR 3.4 to 8.3 ) & 0.69 \\
\hline Change over 12 weeks & $-2.1 \pm 0.6$ & $-0.7 \pm 0.5$ & 0.08 \\
\hline Difference within groups (P-value) & 0.003 & 0.43 & \\
\hline \multicolumn{4}{|l|}{ Intima-media thickness (IMT) (mm) } \\
\hline Baseline (MF $n=19 ;$ PL $n=20$ ) & 0.53 (IQR 0.48 to 0.55 ) & 0.51 (IQR 0.48 to 0.56 ) & 0.75 \\
\hline At 12 weeks & 0.53 (IQR 0.48 to 57 ) & 0.54 (IQR 0.50 to 0.60 ) & 0.67 \\
\hline Change over 12 weeks & $-0.02 \pm 0.08$ & $0.02 \pm 0.05$ & 0.049 \\
\hline Difference within groups (P-value) & 0.62 & 0.04 & \\
\hline \multicolumn{4}{|l|}{ Lysis time (sec) } \\
\hline Baseline (MF $n=13$; PL $n=16$ ) & 342.0 (IQR 267.0 to 393.0 ) & $357.0(\mathrm{IQR} 285.0$ to 400.5$)$ & 0.43 \\
\hline At 12 weeks & 300 (IQR 234 to 324) & 360 (IQR 313 to 632 ) & 0.001 \\
\hline Change over 12 weeks & $-48.0(\mathrm{IQR}-111.0$ to 3.0$)$ & 33.0 (IQR -13.5 to 192.0$)$ & 0.004 \\
\hline Difference within groups (P-value) & 0.03 & 0.06 & \\
\hline \multicolumn{4}{|c|}{ Bone mineral density (BMD) - Total hip (g/cm2) } \\
\hline Baseline (MF $n=16$; PL $n=21)$ & $1.002 \pm 0.150$ & $0.977 \pm 0.181$ & 0.66 \\
\hline At 12 weeks & $1.021 \pm 0.148$ & $0.974 \pm 0.179$ & 0.40 \\
\hline Change over 12 weeks & 0.014 (IQR 0.002 to 0.027 ) & -0.008 (IQR -0.015 to 0.010$)$ & 0.005 \\
\hline Difference within groups (P-value) & 0.02 & 0.44 & \\
\hline \multicolumn{4}{|l|}{$F G F 21(p g / m L)$} \\
\hline Baseline fasting $M F n=19 ;$ PL $n=19$ & 53.1 (IQR 29.9 to 154.6$)$ & 118.9 (IQR 68.8 to 268.6 ) & 0.06 \\
\hline At 12 weeks & 77.0 (IQR 40.0 to 208.0 ) & 34.1 (IQR 18.3 to 147.0 ) & 0.05 \\
\hline
\end{tabular}

Page 25 of 37 


\begin{tabular}{|c|c|c|c|}
\hline Change over 12 weeks & $7.6(\mathrm{IQR}-30.5$ to 169.0$)$ & -78.5 (IQR -234.4 to -1.13$)$ & 0.03 \\
\hline Difference within groups (P-value) & 0.43 & 0.03 & \\
\hline \%change over 12 weeks & $5.0(\mathrm{IQR}-23.6$ to 252.1$)$ & $-66.4(\mathrm{IQR}-87.3$ to -5.0$)$ & 0.01 \\
\hline Maximum post-challenge increment from fasting (change over 12 weeks) $(M F n=18 ; P L n=18$ ) & $167.2(\mathrm{IQR}-124.4$ to 483.7$)$ & $109.2(\mathrm{IQR}-291$ to 1067$)$ & 0.89 \\
\hline \multicolumn{4}{|l|}{ Adiponectin $(\mathrm{ng} / \mathrm{mL})$} \\
\hline Baseline (MF $n=19, P L n=21$ ) & 15.0 (IQR 9.1 to 29.7 ) & 24.1 (IQR 12.8 to 49.5 ) & 0.18 \\
\hline At 12 weeks & 44.9 (IQR 22.7 to 78.1$)$ & 15.9 (IQR 7.3 to 39.7 ) & 0.02 \\
\hline Change over 12 weeks & $27.6 \pm 36.0$ & $-5.5 \pm 32.2$ & 0.004 \\
\hline Difference within groups (P-value) & 0.004 & 0.14 & \\
\hline \multicolumn{4}{|l|}{ Global disease activity VAS (mm) - Rheumatology cohort (pre-specified) } \\
\hline Baseline (MF $n=11 ;$ PL $n=11$ ) & 48 (IQR 28 to 74 ) & 68 (IQR 53 to 80 ) & 0.34 \\
\hline At 12 weeks & $32 \pm 27$ & $57 \pm 23$ & 0.03 \\
\hline Change over 12 weeks & $-17 \pm 22$ & $-4 \pm 32$ & 0.27 \\
\hline Difference within groups (P-value) & 0.02 & 0.69 & \\
\hline
\end{tabular}

Page 26 of 37 
Table 3. Safety evaluation

\begin{tabular}{|c|c|c|c|}
\hline & Metformin & Placebo & P-value \\
\hline \multicolumn{4}{|l|}{ Adverse events (AE) - numbers of episodes reported } \\
\hline Nausea & 19 & 12 & 0.13 \\
\hline Vomiting & 8 & 4 & 0.22 \\
\hline Diarrhoea $^{\wedge}$ & 18 & 8 & 0.01 \\
\hline Flatulence & 4 & 2 & 0.57 \\
\hline Abdominal discomfort & 14 & 12 & 0.78 \\
\hline Constipation & 1 & 3 & 0.49 \\
\hline Indigestion & 4 & 3 & 0.99 \\
\hline Exacerbation of asthma & 8 & 7 & 0.99 \\
\hline Pneumonia $^{+}$ & 1 & 7 & 0.01 \\
\hline Exacerbation of bronchiectasis & 1 & 0 & 0.99 \\
\hline Upper airway viral illness & 6 & 3 & 0.35 \\
\hline Gastrointestinal viral illness & 2 & 0 & 0.33 \\
\hline Candidosis & 0 & 2 & 0.33 \\
\hline Herpes zoster & 0 & 1 & 0.99 \\
\hline Ear infection & 0 & 1 & 0.99 \\
\hline Diverticulitis & 0 & 1 & 0.99 \\
\hline Dental issue (root canal) & 1 & 0 & 0.99 \\
\hline Ulcer & 2 & 1 & 0.99 \\
\hline Dysglycaemia/osmotic symptoms & 5 & 11 & 0.08 \\
\hline Ischemic heart disease/Atrial fibrillation/atypical chest pain ${ }^{\#}$ & 2 & 2 & 0.99 \\
\hline B12 deficiency & 0 & 2 & 0.33 \\
\hline $\begin{array}{l}\text { Other (affecting <4\% patients: fall, car incident, incidental imaging } \\
\text { findings, dog bite, schiatica, headache, hematoma etc.) }\end{array}$ & 14 & 12 & 0.78 \\
\hline \multicolumn{4}{|l|}{ Serious adverse events $(\mathrm{SAE}) *$ - numbers of episodes reported } \\
\hline Exacerbation of asthma & 0 & 3 & 0.10 \\
\hline Pneumonia & 0 & 1 & 0.99 \\
\hline Diverticulitis & 0 & 1 & 0.99 \\
\hline Ischemic heart disease & 1 & 0 & 0.99 \\
\hline Atypical chest pain & 0 & 1 & 0.99 \\
\hline Severe Raynaud's & 0 & 1 & 0.99 \\
\hline Severe osmotic symptoms & 0 & 2 & 0.33 \\
\hline All SAES & 1 & 9 & 0.001 \\
\hline
\end{tabular}

AE and SAE were defined and reported as per Good Clinical Practice: Here SAE equated to inpatient hospitalizations ( $\geq 24 \mathrm{~h}$ stay). The degree of severity was assessed separately to the definition of SAE. The study drugs were not deemed causative of the SAE. The reporting period ended 30 days post cessation of treatment.

A $10(38 \%)$ metformin-treated patients compared to $2(7 \%)$ on placebo reported diarrhoea during the first 4 weeks after treatment initiation ( $\mathrm{p}=0.009)$ with a median symptom resolution of 3 days (IQR 2 to 28) in the metformin cohort and no between-group difference in diarrhoea reports during subsequent follow-ups.

${ }^{+}$The total number of infective episodes was not significantly different between the treatment groups (15 on metformin vs. 21 on placebo, $\left.\mathrm{p}=0.24\right)$, but their severity differed. There were 2 moderate or severe infective episodes on metformin compared to 11 on placebo ( $\mathrm{p}=0.001$ ); the incidence of mild 
infective episodes was similar ( 13 on metformin compared to 10 on placebo, $\mathrm{p}=0.56$ ). This suggested $33 \%$ ( $95 \% \mathrm{CI} 12$ to 54 ) absolute risk reduction of moderate and severe infective events on metformin $(\mathrm{p}=0.009)$ where the number needed to treat was 3 (95\%CI 2 to 8 ).

- During hospital encounters and admissions, glycaemia was monitored and managed as per routine clinical practice, independently of the study. Apart from the two subjects developing severe osmotic symptoms, withdrawn from the study, no other patient was deemed to require a glucose-lowering pharmacological intervention.

${ }^{\#}$ Ischemic heart disease diagnosed incidentally in an elderly heavy smoker ( $>40$ pack year smoking history) treated with a coronary stenting off the investigational medicinal product.

*There were less serious adverse events affecting the respiratory system in the metformin arm $(\mathrm{p}=0.03)$. The number of episodes of asthma exacerbations was similar; out of these, the proportional rate of severe episodes seemed higher on placebo $(\mathrm{p}=0.08)$. Overall, there was a $30 \%(95 \% \mathrm{CI} 10$ to 49$)$ absolute risk reduction of serious adverse events on metformin $(\mathrm{p}=0.01)$ which suggests about 3 ( $95 \%$ CI 2 to 10$)$ patients needing treatment to prevent 1 hospital admission. 


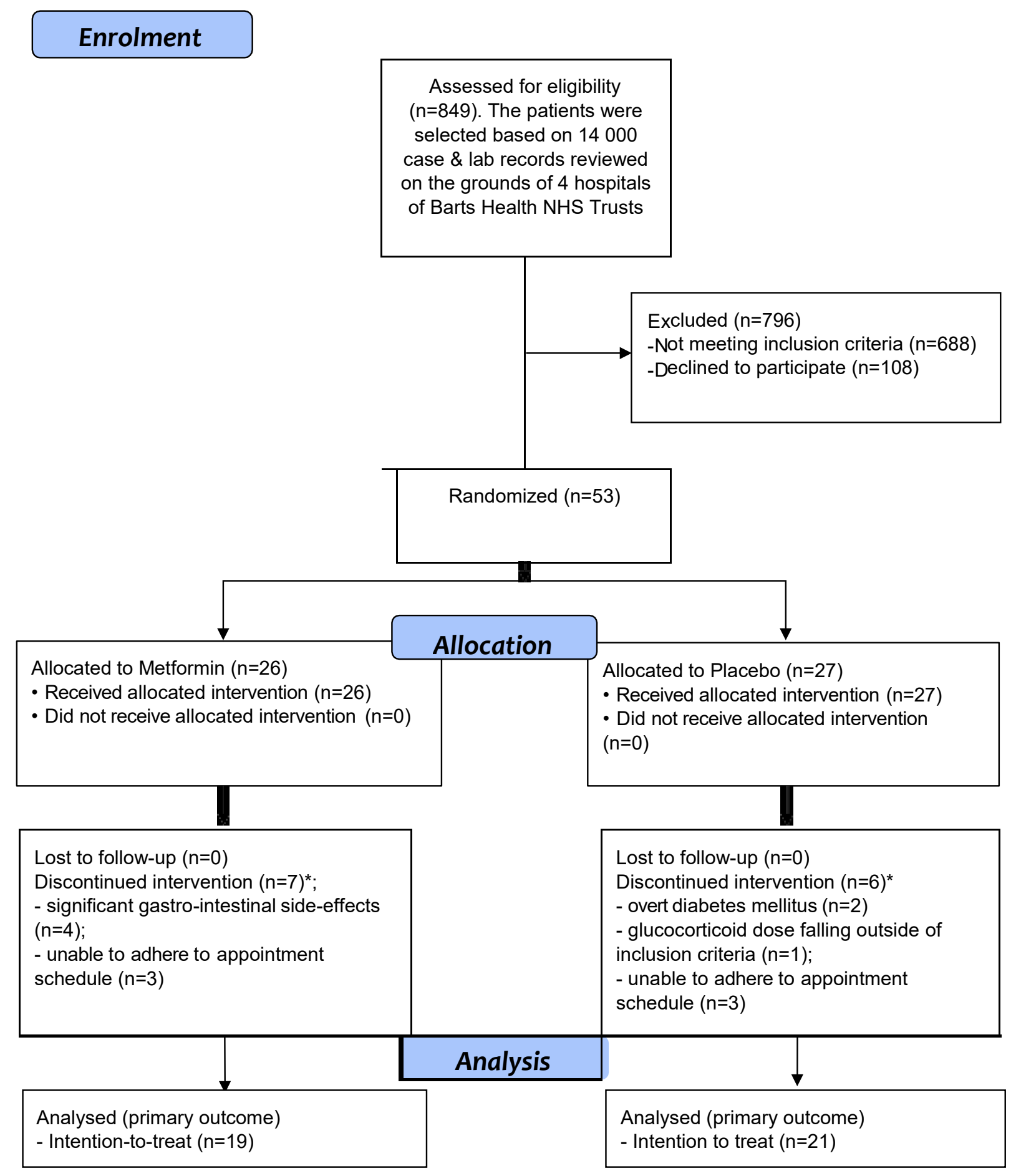

*Drop-out rates were not different in the metformin and placebo groups.

Figure 1. Trial flow diagram 
a
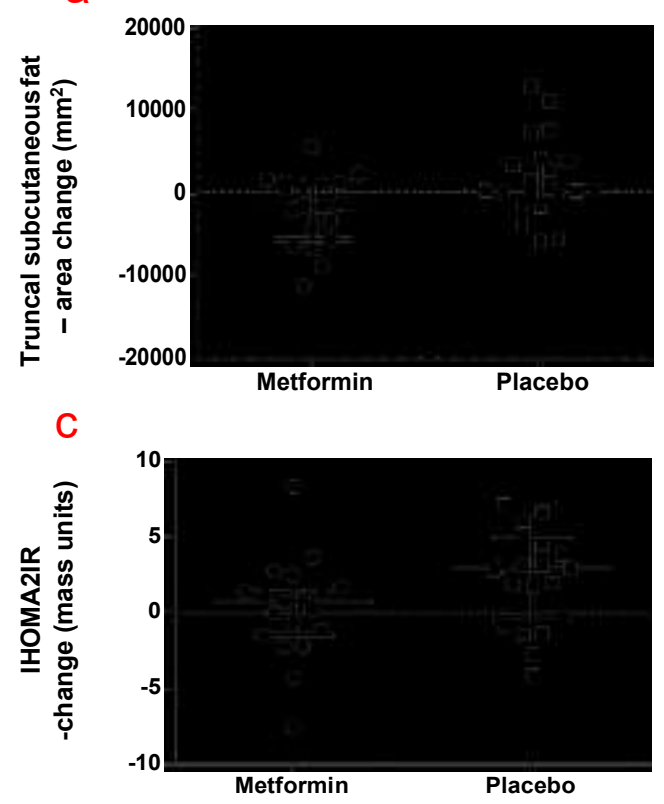

e

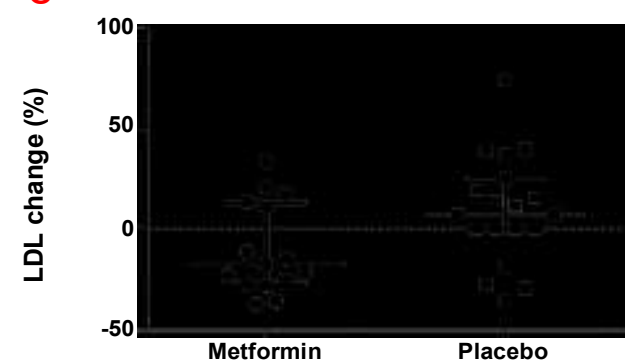

g

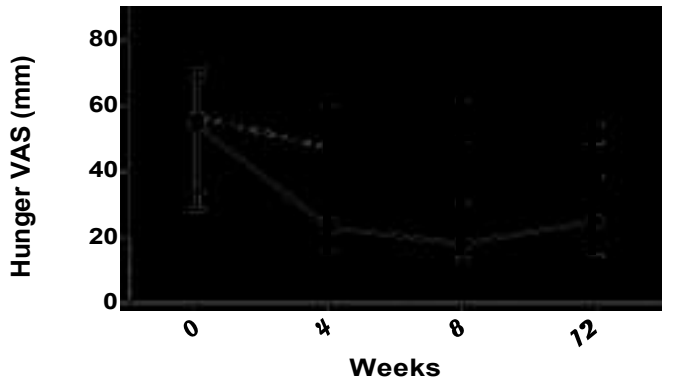

Metformin b

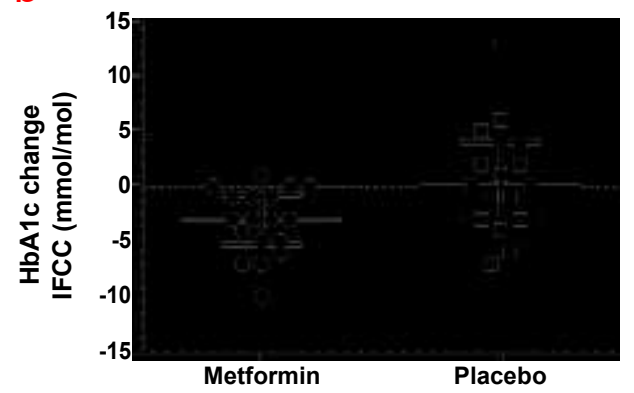

d

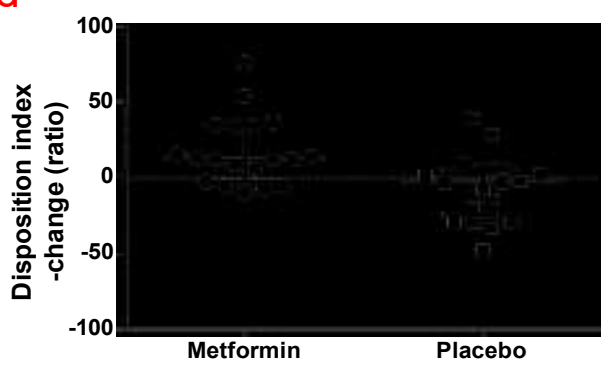

f

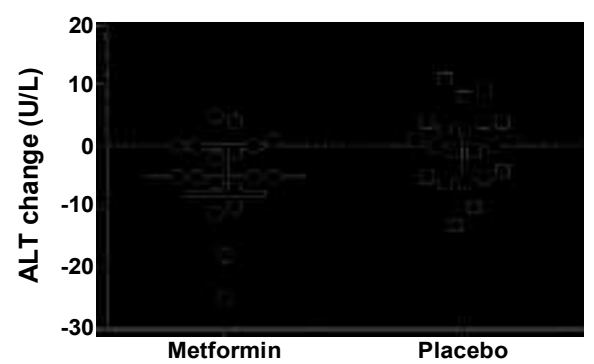

$\mathrm{h}$

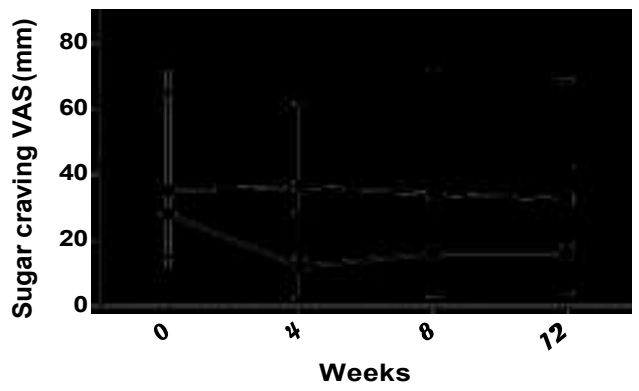

Placebo

\section{Figure 2. Metformin effects on appetite, pancreas, fat and liver}

Figure 2. legend: Changes over 12 weeks between metformin- and placebo-treated groups in a) truncal subcutaneous fat area $(\mathrm{p}=0.01), \mathrm{b}) \mathrm{HbA} 1 \mathrm{c}(\mathrm{p}=0.007), \mathrm{c})$ insulin resistance $(\mathrm{p}=0.04), \mathrm{d})$ HOMA2\%B/HOMA2IR disposition index $(\mathrm{p}=0.001)$, e) LDL in statin-naïve patients $(\mathrm{p}=0.03), \mathrm{f}) \operatorname{ALT}(\mathrm{p}=0.03)$, g) hunger $(\mathrm{p}=0.04)$ and $\mathrm{h})$ sugar craving $(\mathrm{p}=0.01)$ by $100 \mathrm{~mm}$ 
visual analogue scales (VAS). Data are presented as median and interquartile range; data points (a-f) represent individual patients. P-values were assessed by T-test/Mann-Whitney (a-f), repeated-measures two-way ANOVA (g, h).

Page 31 of 37 
a

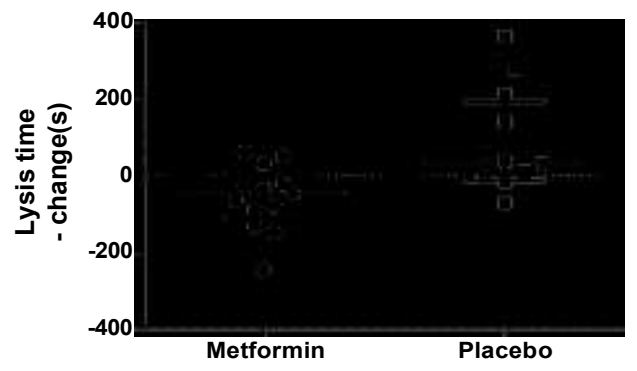

C

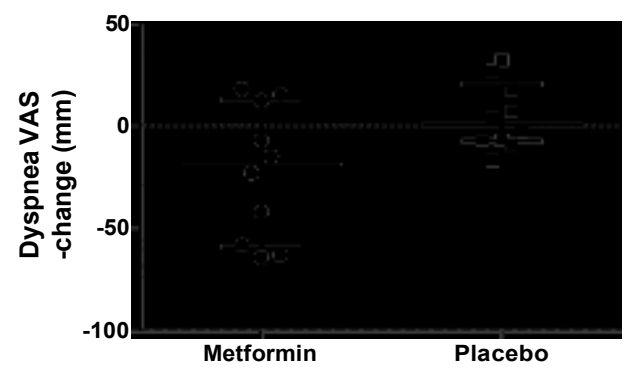

e

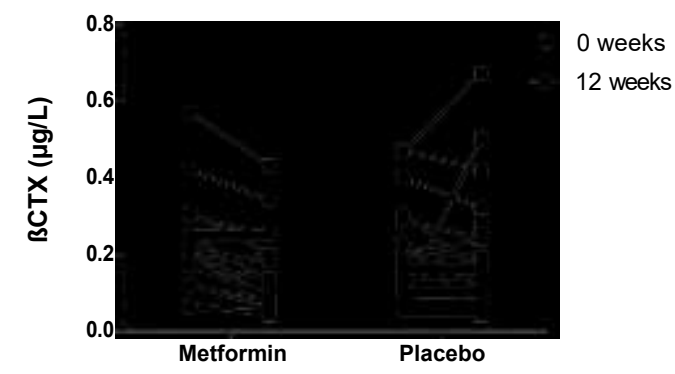

b

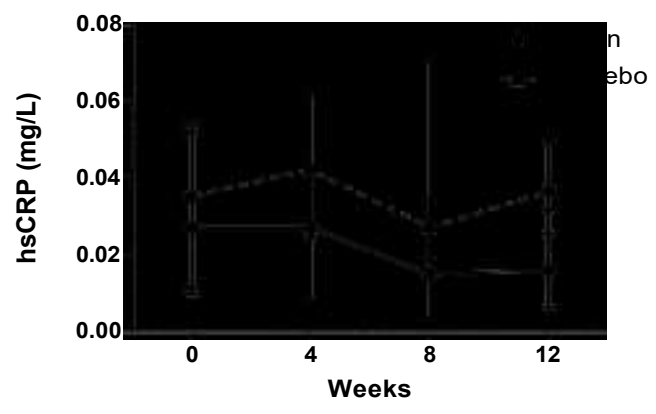

d

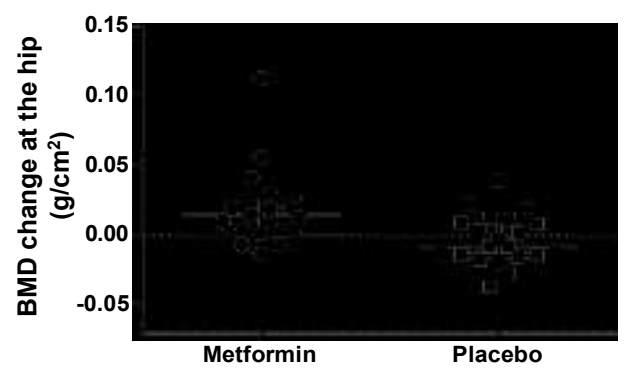

f

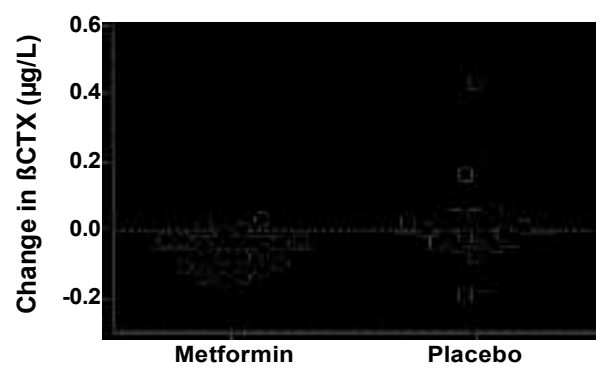

Figure 3. Metformin effects on fibrinolysis, inflammation and bone metabolism

Figure 3 legend: Changes over 12 weeks between treatment groups in a) lysis time $(p=0.004), b)$ hsCRP $(p=0.04), c)$ dyspnoea $100 \mathrm{~mm}$ VAS in patients with diseases affecting the respiratory system $(\mathrm{p}=0.03), \mathrm{d})$ BMD at the hip $(\mathrm{p}=0.005)$, e) $\beta C T X$ showing individual patient data: changes within-metformin group $(\mathrm{p}=0.001)$; changes within-placebo group $(p=0.82), f) \beta C T X$ : between-group difference $(p=0.01)$. Medians with interquartile error bars are presented $(a-d, f)$; data points (a, c, d, e, f) represent individual patients. P-values were assessed by T-test/Mann-Whitney (a, c-f) and repeatedmeasures two-way ANOVA (b).

hsCRP, high sensitivity $\mathrm{C}$-reactive protein; VAS, visual analogue scale; $\beta \mathrm{CTX}, \beta$-C-terminal telopeptide; $\mathrm{BMD}$, bone mineral density 


\section{Supporting information}

Supplementary Material text, tables and figures

CONSORT checklist

Study protocol

\section{References}

1. Couvaras L, Trijau S, Delamotte G, Pradel V, Pham T, Lafforgue P. [Epidemiological description of long-term oral glucocorticoid use: Results from the French health insurance database]. Rev Med Interne 2018; 39: $777-81$.

2. Laugesen K, Jorgensen JOL, Petersen I, Sorensen HT. Fifteen-year nationwide trends in systemic glucocorticoid drug use in Denmark. Eur J Endocrinol 2019.

3. Pivonello R, Isidori AM, De Martino MC, Newell-Price J, Biller BM, Colao A. Complications of Cushing's syndrome: state of the art. Lancet Diabetes Endocrinol 2016; 4: 611-29.

4. Spivey CA, Griffith J, Kaplan C, Postlethwaite A, Ganguli A, Wang J. A Retrospective Analysis of Corticosteroid Utilization Before Initiation of Biologic DMARDs Among Patients with Rheumatoid Arthritis in the United States. Rheumatol Ther 2018; 5: 255-70.

5. Pivonello R, Faggiano A, Lombardi G, Colao A. The metabolic syndrome and cardiovascular risk in Cushing's syndrome. Endocrinol Metab Clin North Am 2005; 34: 327-39, viii.

6. Souverein PC, Berard A, Van Staa TP, et al. Use of oral glucocorticoids and risk of cardiovascular and cerebrovascular disease in a population based case-control study. Heart 2004; 90: 859-65.

7. Pernicova I, Korbonits M. Metformin--mode of action and clinical implications for diabetes and cancer. Nat Rev Endocrinol 2014; 10: 143-56.

8. Kola B, Christ-Crain M, Lolli F, et al. Changes in adenosine 5'-monophosphate-activated protein kinase as a mechanism of visceral obesity in Cushing's syndrome. J Clin Endocrinol Metab 2008; 93: 4969-73. 
9. Christ-Crain M, Kola B, Lolli F, et al. AMP-activated protein kinase mediates glucocorticoid-induced metabolic changes: a novel mechanism in Cushing's syndrome. FASEB J 2008; 22: 1672-83.

10. Seelig E, Meyer S, Timper K, et al. Metformin prevents metabolic side effects during systemic glucocorticoid treatment. Eur J Endocrinol 2017; 176: 349-58.

11. Rockall AG, Sohaib SA, Evans D, et al. Computed tomography assessment of fat distribution in male and female patients with Cushing's syndrome. Eur J Endocrinol 2003; 149: 561-67.

12. Kaess BM, Pedley A, Massaro JM, Murabito J, Hoffmann U, Fox CS. The ratio of visceral to subcutaneous fat, a metric of body fat distribution, is a unique correlate of cardiometabolic risk. Diabetologia 2012; 55: 2622-30.

13. Wallace TM, Levy JC, Matthews DR. Use and abuse of HOMA modeling. Diabetes Care 2004; 27 : $1487-95$.

14. Stunkard AJ, Messick S. The three-factor eating questionnaire to measure dietary restraint, disinhibition and hunger. J Psychosom Res 1985; 29: 71-83.

15. Doherty P, Harrison AS, Knapton M, Dale V. Observational study of the relationship between volume and outcomes using data from the National Audit of Cardiac Rehabilitation. Open Heart 2015; 2: e000304.

16. Umpierrez GE, Hellman R, Korytkowski MT, et al. Management of hyperglycemia in hospitalized patients in non-critical care setting: an endocrine society clinical practice guideline. J Clin Endocrinol Metab 2012; 97: 16-38.

17. Geer EB, Shen W, Gallagher D, et al. MRI assessment of lean and adipose tissue distribution in female patients with Cushing's disease. Clin Endocrinol (Oxf) 2010; 73: 469-75.

18. Fox CS, Massaro JM, Hoffmann U, et al. Abdominal visceral and subcutaneous adipose tissue compartments: association with metabolic risk factors in the Framingham Heart Study. Circulation 2007; 116: $39-48$.

19. Luo T, Nocon A, Fry J, et al. AMPK Activation by Metformin Suppresses Abnormal Extracellular Matrix Remodeling in Adipose Tissue and Ameliorates Insulin Resistance in Obesity. Diabetes 2016; 65: 2295 310.

Page 34 of 37 
20. Iozzo P, Hallsten K, Oikonen V, et al. Effects of metformin and rosiglitazone monotherapy on insulinmediated hepatic glucose uptake and their relation to visceral fat in type 2 diabetes. Diabetes Care 2003; 26: 2069-74.

21. Gore DC, Wolf SE, Sanford A, Herndon DN, Wolfe RR. Influence of metformin on glucose intolerance and muscle catabolism following severe burn injury. Ann Surg 2005; 241: 334-42.

22. Anderson AJ, Andrew R, Homer NZ, et al. Metformin Increases Cortisol Regeneration by 11betaHSD1 in Obese Men With and Without Type 2 Diabetes Mellitus. J Clin Endocrinol Metab 2016; 101: 3787-93.

23. Knowler WC, Barrett-Connor E, Fowler SE, et al. Reduction in the incidence of type 2 diabetes with lifestyle intervention or metformin. N Engl J Med 2002; 346: 393-403.

24. Krakoff J, Clark JM, Crandall JP, et al. Effects of metformin and weight loss on serum alanine aminotransferase activity in the diabetes prevention program. Obesity (Silver Spring) 2010; 18: 1762-67.

25. Burt MG, Roberts GW, Aguilar-Loza NR, Frith P, Stranks SN. Continuous monitoring of circadian glycemic patterns in patients receiving prednisolone for COPD. J Clin Endocrinol Metab 2011; 96: 1789-96.

26. Levitan EB, Song Y, Ford ES, Liu S. Is nondiabetic hyperglycemia a risk factor for cardiovascular disease? A meta-analysis of prospective studies. Arch Intern Med 2004; 164: 2147-55.

27. Feingold KR, Grunfeld C. The Effect of Inflammation and Infection on Lipids and Lipoproteins. In: De Groot LJ, Chrousos G, Dungan K, et al., eds. Endotext. South Dartmouth (MA); 2015: https://www.ncbi.nlm.nih.gov/pubmed/26561701.

28. Tataranni PA, Larson DE, Snitker S, Young JB, Flatt JP, Ravussin E. Effects of glucocorticoids on energy metabolism and food intake in humans. Am J Physiol 1996; 271: E317-25.

29. Dushay JR, Toschi E, Mitten EK, Fisher FM, Herman MA, Maratos-Flier E. Fructose ingestion acutely stimulates circulating FGF21 levels in humans. MolMetab 2015; 4: 51-57.

30. van ZB, Nur E, Squizzato A, et al. Systematic review on the effect of glucocorticoid use on procoagulant, anti-coagulant and fibrinolytic factors. J Thromb Haemost 2010; 8: 2483-93.

31. Sumaya W, Wallentin L, James SK, et al. Fibrin clot properties independently predict adverse clinical outcome following acute coronary syndrome: a PLATO substudy. Eur Heart J 2018; 39: 1078-85. 
32. Grant PJ. Beneficial effects of metformin on haemostasis and vascular function in man. Diabetes Metab 2003; 29: 6S44-52.

33. Meaney E, Vela A, Samaniego V, et al. Metformin, arterial function, intima-media thickness and nitroxidation in metabolic syndrome: the mefisto study. Clin Exp Pharmacol Physiol 2008; 35: 895-903.

34. Bjornstad P, Schafer M, Truong U, et al. Metformin Improves Insulin Sensitivity and Vascular Health in Youth With Type 1 Diabetes Mellitus. Circulation 2018; 138: 2895-907.

35. Preiss D, Lloyd SM, Ford I, et al. Metformin for non-diabetic patients with coronary heart disease (the CAMERA study): a randomised controlled trial. Lancet Diabetes Endocrinol 2014; 2: 116-24.

36. Cameron AR, Morrison VL, Levin D, et al. Anti-Inflammatory Effects of Metformin Irrespective of Diabetes Status. Circ Res 2016; 119: 652-65.

37. Rogliani P, Ora J, Di Daniele N, Lauro D. Pleiotropic effects of hypoglycemic agents: implications in asthma and COPD. Curr Opin Pharmacol 2018; 40: 34-38.

38. Mor A, Petersen I, Sorensen HT, Thomsen RW. Metformin and other glucose-lowering drug initiation and rates of community-based antibiotic use and hospital-treated infections in patients with type 2 diabetes: a Danish nationwide population-based cohort study. BMJ Open 2016; 6: e011523.

39. Freckelton J, Evans JA, Croagh D, Moore GT. Metformin use in diabetics with diverticular disease is associated with reduced incidence of diverticulitis. Scand J Gastroenterol 2017; 52: 969-72.

40. Ladson G, Dodson WC, Sweet SD, et al. The effects of metformin with lifestyle therapy in polycystic ovary syndrome: a randomized double-blind study. Fertil Steril 2011; 95: 1059-66.

41. Kuusalo L, Puolakka K, Kautiainen H, et al. High burden of adverse events is associated with reduced remission rates in early rheumatoid arthritis. Clin Rheumatol 2018; 37: 1689-94.

42. Isidori AM, Venneri MA, Graziadio C, et al. Effect of once-daily, modified-release hydrocortisone versus standard glucocorticoid therapy on metabolism and innate immunity in patients with adrenal insufficiency (DREAM): a single-blind, randomised controlled trial. Lancet Diabetes Endocrinol 2018; 6: 173-85.

43. Zhao J, Li Y, Zhang H, et al. Preventative effects of metformin on glucocorticoid-induced osteoporosis in rats. J Bone Miner Metab 2019; 37: 805-14. 
44. Vestergaard P, Rejnmark L, Mosekilde L. Relative fracture risk in patients with diabetes mellitus, and the impact of insulin and oral antidiabetic medication on relative fracture risk. Diabetologia 2005; 48: 1292-99. 45. Naka KK, Kalantaridou SN, Kravariti M, et al. Effect of the insulin sensitizers metformin and pioglitazone on endothelial function in young women with polycystic ovary syndrome: a prospective randomized study. Fertil Steril 2011; 95: 203-9.

46. Giles JT, Allison M, Blumenthal RS, et al. Abdominal adiposity in rheumatoid arthritis: association with cardiometabolic risk factors and disease characteristics. Arthritis Rheum 2010; 62: 3173-82.

47. Hadigan C, Corcoran C, Basgoz N, Davis B, Sax P, Grinspoon S. Metformin in the treatment of HIV lipodystrophy syndrome: A randomized controlled trial. JAMA 2000; 284: 472-77.

48. Lord J, Thomas R, Fox B, Acharya U, Wilkin T. The effect of metformin on fat distribution and the metabolic syndrome in women with polycystic ovary syndrome--a randomised, double-blind, placebocontrolled trial. BJOG 2006; 113: 817-24. 